\title{
Primary Negative Symptoms: Appraisal of a Misty Outlook
}

\author{
Saeed Shoja Shafti* \\ Department of Psychiatry, University of Social Welfare and Rehabilitation Sciences, Iran
}

*Corresponding author: Saeed Shoja Shafti, Professor of Psychiatry, University of Social Welfare and Rehabilitation Sciences (USWR), Razi Psychiatric Hospital, Tehran, Iran.

To Cite This Article: Saeed Shoja Shafti. Primary Negative Symptoms: Appraisal of a Misty Outlook. Am J Biomed Sci \& Res. 2019 - 2(2). AJBSR. MS.ID.000573. DOI: 10.34297/AJBSR.2019.02.000573

Received: February 06, 2019 | Published: March 28, 2019

\begin{abstract}
Summary
Negative symptoms, which are among the main criteria in DSM-5 for diagnosis of schizophrenia, are categorized by some of the researchers into 'primary vs. secondary', 'enduring vs. transitory' and 'treatment-resistant vs. treatment-responsive' groups. Besides, adjunctive strategies are desperately acknowledged as the necessary complementary inventions in this regard.

But according to a quantity of data, such invented classification into above-mentioned clusters, may not be verified reasonably. In addition, unconditional pessimistic standpoint regarding inflexibility of 'primary negative symptoms', against therapeutic approaches, does not seem to be tenable and thus needs to be revised. So, the time has arrived for a critical review about such a conjectural dichotomy, which is seeking diligently cure for no authentic theme.
\end{abstract}

\section{Introduction}

According to DSM-5 and for diagnosis of schizophrenia:" .... At least two Criterion A symptoms must be present for a significant portion of time during a 1-month period or longer. At least one of these symptoms must be the clear presence of delusions (Criterion Al), hallucinations (Criterion A2), or disorganized speech (Criterion A3). Grossly disorganized or catatonic behavior (Criterion A4) and negative symptoms (Criterion A5) may also be present. Schizophrenia involves impairment in one or more major areas of functioning (Criterion B). Avolition (i.e., reduced drive to pursue goal-directed behavior; Criterion A5) is linked to the social dysfunction described under Criterion B. There is also strong evidence for a relationship between cognitive impairment and functional impairment in individuals with schizophrenia. Some signs of the disturbance must persist for a continuous period of at least 6 months (Criterion C). Prodromal symptoms often precede the active phase, and residual symptoms may follow it, characterized by mild or sub-threshold forms of hallucinations or delusions. Negative symptoms are common in the prodromal and residual phases and can be severe. Individuals who had been socially active may become withdrawn from previous routines. Such behaviors are often the first sign of a disorder...." “.... Negative symptoms account for a substantial portion of the morbidity associated with schizophrenia but are less prominent in other psychotic disorders. Two negative symptoms are particularly prominent in schizophrenia: diminished emotional expression and avolition. Diminished emotional expression includes reductions in the expression of emotions in the face, eye contact, intonation of speech (prosody), and movements of the hand, head, and face that normally give an emotional emphasis to speech.

Avolition is a decrease in motivated self-initiated purposeful activities. The individual may sit for long periods of time and show little interest in participating in work or social activities. Other negative symptoms include alogia, anhedonia, and asociality. Alogia is manifested by diminished speech output. Anhedonia is the decreased ability to experience pleasure from positive stimuli or degradation in the recollection of pleasure previously experienced. Asociality refers to the apparent lack of interest in social interactions and may be associated with avolition, but it can also be a manifestation of limited opportunities for social interactions......" [1]. So again, negative symptoms in schizophrenia, as one of the main criteria in DSM-5, include:
a. Restricted up to flat affect,
b. Apathy,
c. Alogia, 

d. Anhedonia,
e. Avolition,
f. A sociality, and lastly
g. Attention Deficit [2].

Previously in DSMIV-TR, existence of at least two of these symptoms for at least twelve months was enough for diagnosis of deficit schizophrenia [3].

Anxiety, suspiciousness, mental retardation, depression, drug induced parkinsonism, active positive symptoms (like delusion and hallucination) and lack of environmental stimulants and so on are regarded as potential variables that can cause secondary negative symptoms or reinforcement of primary ones. Among them Affective Blunting, Alogia and Attention Deficit are grouped by some of the researchers as enduring, deficit, persistent, trait and treatmentresistant cluster and Anhedonia, Asociality, Avolition and Apathy as non-enduring, phasic, transitory, state and treatment-responsive cluster of negative symptoms. [4,5]. Hence, it is supposed that the first group is due to structural and developmental factors and the second cluster is caused by imbalance of dopamine in the mesolimbic-mesocortical circuit. In addition, the first group is usually more evident in the residual or pre-morbid phase of schizophrenia, while the second group is more observable in the acute (psychotic) phase and pre and/or post psychotic illness. Besides, the first cluster is recognized as poor prognostic sign, while the later one as either independent from prognosis or as an indication of good prognosis.

Moreover, the enduring group seems to be more prevalent among the patient's relatives in comparing with the non-enduring group. Even among the enduring cluster, some researchers believe that Affective blunting or flattening and poverty of speech, perhaps, are more appropriate for including as 'primary negative symptoms' and they consider them in relationship with deterioration of social function during puberty, while Attention impairment and poverty of content of speech are perceived in relationship with cognitive disturbance and poor school performance of such patients in the former period of their illness. Neither of the aforesaid functions seems to have any association with symptoms of second cluster, like Avolition-Apathy or Anhedonia-Asociality [4,5]. Disregard to validity of such kind of classification or viewpoints with respect to clinical aspects of primary negative symptoms, also a general despair is evident regarding resistance of 'primary negative symptoms' against antipsychotic treatment. Therefore, adjunctive strategies are commonly acknowledged as the complementary inventions in this regard [6]. Cognitive deficits of schizophrenic patients too, do not seem to be independent totally from 'primary negative symptoms [5]. But according to a quantity of data, such invented categorization into 'enduring vs. transient', and 'primary vs. secondary' negative symptoms, may not be verified reasonably.

For example, in a series of double-blind clinical trials, we tried to assess the effectiveness of different adjunctive drugs for improvement of apparently 'primary negative symptoms', after employment of a specific inclusion and exclusion criteria, which was determined according to the Diagnostic and Statistical Manual of Mental Disorders,4th edition, Text Revision. In this regard and in the first tryout on 40 schizophrenic cases, we found that citalopram, alprazolam, and clomipramine, in minimum to moderate doses, were significantly more effective than placebo $(\mathrm{P}=0.001, \mathrm{P}=0.01$ and $\mathrm{P}=0.01$, respectively) in reducing the score of SANS for different items, though commonly about $20 \%$ in comparison with the baseline. Also, there was not any direct relationship between response and severity of the negative symptoms [7]. It is mentionable that in this evaluation, Affective Blunting showed the most and Anhedonia-A sociality the least improvement.

Consequently, and in a further study, the effectiveness of bromocripme, fluoxetine and nortiptyline were judged against placebo in 100 schizophrenia patients. According to the results of this experiment, while all of the used adjunctive drugs, in minimum to moderate doses, were better than placebo, only nortriptyline was significantly more effective $(\mathrm{P}=0.005)$ [8]. In this trial, Attention Deficit showed the most and Affecting Blunting the least improvement by adjunctive drugs. Once more, there was no clear relationship between severity of the symptoms and their further response.

In another trial, the effectiveness of maprotiline and fluvoxamine were compared to placebo, in 30 schizophrenic patients, and according to the results, both of them were more effective than placebo, though it was significant only with respect to maprotiline $(\mathrm{P}=0.01)$. In this sample severe symptoms responded more than mild ones to adjunctive drugs $[9,10]$.

Therefore, according to our findings, Affective Blunting and Attention Deficit in the first and second trials, respectively, were the most responsive negative symptoms; and again, Affective Blunting together with Alogia and Anhedonia-A sociality were the most responsive ones in the third trial. Totally, in $31.2 \%, 28 \%, 26.4 \%$, $24 \%$ and $22.4 \%$ of the patients there was around $20 \%$ reduction in the severity of Attention Deficit, Alogia, Affective Blunting, Anhedonia-A sociality and finally Avolition-Apathy, respectively.

Based on the exclusion criteria, therefore all the negative symptoms in those samples could be supposed as primary ones, and as has been said before, Affective Blunting in the first trial, Attention Deficit in the second trial, and Affective Blunting, Alogia and Anhedonia-A sociality in the third trial showed the best response to adjunctive drugs. So, in contrast to the old-fashioned classification, symptoms like Avolition-Apathy in the third trial and Anhedonia-A sociality in the first and second trials were the least responsive ones. Similar results as well can be found in a lot of studies regarding negative symptoms.

Consequently, the following conclusions can be taken into consideration with respect to our discussion:

a. Unconditional pessimistic standpoint regarding inflexibility of 'primary negative symptoms' against therapeutic approaches does not seem to be tenable and needs to be revised.

b. There is no sufficient evidence for classification of 'primary negative symptoms' into two aforesaid clusters of 
'enduring, treatment-resistant' and 'transient, treatmentresponsive' ones.

c. Also, categorization of negative symptoms into' primary 'and 'secondary' is a challenging issue. Firstly, DFSM-5 has not stressed on precise identification of primary ones for diagnosis of schizophrenia. It seems that it has supposed only one type of negative symptoms that are easily diagnosable. Secondly, positive symptoms are in the realm of psychopathology, while negative symptoms are mainly in the realm of interpersonal function. Function is a multidimensional concept, and its interactional aspect, which is assessed by clinician, can have root in the pre-morbid traits. Cluster A personality disorders, like schizotypal and schizoid, have traits similar to negative symptoms, and while all of them are in the realm of behavior, there is no necessity that always and in all situations they ought to be considered dysfunctional.

d. According to the aforesaid trials, a positive, though restricted, response due to adjunctive treatments was evident in the clinical spectrum of negative symptoms. There are other studies also that have shown that negative symptoms are treatment responsive, $[11,12]$ and there is no necessity to consider it factual only with respect to 'secondary negative symptoms. But if, in opposition to the diagnostic criteria of DSM-5, differentiation between 'primary 'and 'secondary' in the realm of therapy is so sensitive and hard to accomplish, then it is better to consider them totally as' secondary', instead of 'primary', which can only promote a morbid cycle of stigmatization. That kind of deficit symptoms that are due to disturbances of mood, anxiety, positive symptoms, extrapyramidal side effects and so on, are only a cluster of byproducts that can be ruled out by careful clinical exam, and they are not negative symptoms.

e. Proponents of 'primary negative symptoms', who may have based their suppose on the observable facts of 'disturbance of function', 'chronicity' and 'neuro-anatomical changes' of schizophrenic patients, believe in the existence of a set of similar symptoms that are treatment resistant and enduring , not originating from the abovementioned .Hence, they termed the later cluster as 'secondary negative symptoms'. But what kind of treatment resistant is it that easily responds to even the minimum doses of a variety of SSRIs, TCAs and etc. There are a lot of trials in this regard, many of them with valuable scientific methods that have shown treatability of this kind of symptoms.

Although the proponents of primary negative symptoms discard most of them as faulty that are dealing mostly with secondary ones, but it does not look as if their facts are in essence different from other's facts. Whichever subject that cannot be separated, by any means, from the concept of' secondary' perhaps is nothing except than a wholly 'secondary' phenomenon. If the problem is in the apparent reality of "disturbance of function", then that aspect can be described more precisely. If the neuro-anatomical changes bear in mind the Hughlings Jackson's perspective of 'progressive dissolution of function' and subsequent neurological classification of positive and negative symptoms, it should not be ignored that schizophrenia is still in the field of psychiatry. Sticking so rigorously to "primary" vs. "secondary", based on indistinct proofs and subjective inferences, is not truthfully in concord with the fundamental objectives of psychiatric rehabilitation, because promotion of deinstitutionalization is not achievable with endorsement of stigmatization. Consequently, it seems that the time has arrived for a critical review about such a conjectural dichotomy, which is seeking diligently cure for no authentic theme.

\section{References}

1. American Psychiatric Association (2013) Diagnostic and Statistical Manual of Mental Disorders. ( $5^{\text {th }}$ edn)., Washington, DC, USA.

2. Nancy Andreasen, Micheal Falbum (1994) Characteristic symptoms of schizophrenia. DSM IV source Book, Chapter 22: 365-380.

3. American Psychiatric Association (2000) American psychiatric association. Diagnostic and Statistical Manual of Mental Disorders. ( $^{\text {th }}$ edn), Washington, DC, USA.

4. Carpenter WT, Heinrichs DW, Wagman AMI (1988) Deficit and nondeficit forms of schizophrenia: The concept Am J Psychiatry 145(5): 578-583.

5. William T Carpenter, Robert W Buchanan (1991) Brian Kirkpatrick the concept of the negative symptoms of schizophrenia, Negative schizophrenic symptoms. Pathophysiology and Clinical Implications, American Psychiatric Press Washington DC, USA, p. 5-20.

6. Robert W Buchanan, Martin Brandes, Alan Breier (1996) Treating Negative symptoms: pharmacological strategies, the new pharmacotherapy of schizophrenia. American psychiatric press, Washington DC, USA, pp. 179-197.

7. Shoja Shafti S (2003) Effectiveness of Citalopram, Alprazolam and Clomipramine in amelioration of negative symptoms in Schizophrenia. Journal of Rehabilitation Tehran 3(12): 42-49.

8. Shoja Shafti S (2004) Effectiveness of Bromocriptine, Flouxetine and Nortriptyline in Ameliorating the negative symptoms of Schizophrenia. Journal of Rehabilitation Tehran 5(16): 58-63.

9. Shoja Shafti S (2005) Drug -Specific Responsiveness of Negative Symptoms. International Journal of Psychosocial Rehabilitation 10(1): 43-51.

10. Shoja Shafti S (2004) Rehabilitation of Schizophrenia: Adjunctive Therapy of Negative Symptoms. Iranian Rehabilitation Journal (IRJ) $1(2)$.

11. Shoja Shafti S (2007) Amelioration of Negative Symptoms in Schizophrenia by Escitalopram: A Double Blind, Placebo-Controlled Clinical Trial. Clinical Schizophrenia \& Related Psychosis 1(3): 255-258.

12. Shoja Shafti S (2006) Treatment-Responsiveness of Negative Symptoms in Schizophrenia: A double-blind placebo-control clinical trial. Iranian Journal of Medical Sciences 31(3): 135-138. 